\title{
Exhaled IL-8 in Systemic Lupus Erythematosus with and without Pulmonary Fibrosis
}

\author{
Agnieszka Nielepkowicz-Goździńska • Wojciech Fendler • Ewa Robak • \\ Lilianna Kulczycka-Siennicka • Pawel Górski • Tadeusz Pietras • \\ Ewa Brzeziańska • Adam Antczak
}

Received: 17 December 2012/ Accepted: 9 October 2013/Published online: 4 February 2014

(C) The Author(s) 2014. This article is published with open access at Springerlink.com

\begin{abstract}
The purpose of this study is to evaluate the relationship between the concentration of interleukin-8 (IL8 ) in exhaled breath condensate (EBC) and bronchoalveolar lavage fluid (BALF) with the disease activity score and pulmonary function of systemic lupus erythematosus (SLE) patients with and without pulmonary fibrosis. Thirty-four SLE patients and 31 healthy controls were enrolled and evaluated using high-resolution computed tomography (HRCT), pulmonary function tests, systemic lupus activity measure (SLAM), assessing BALF and EBC. IL-8 levels in BALF and EBC samples were measured with an enzymeimmunosorbent assay kit. The mean ( \pm SEM) IL-8 concentrations in BALF and EBC were higher in SLE patients compared to healthy controls (34.84 \pm 95.0 vs. $7.65 \pm 21.22 \mathrm{pg} / \mathrm{ml}, \quad p<0.001 ; 3.82 \pm 0.52 \mathrm{pg} / \mathrm{m} \quad$ vs. $1.7 \pm 1.7 \mathrm{pg} / \mathrm{ml}, p<0.001$, respectively). SLE patients had increased percentage of neutrophils in BALF when
\end{abstract}

A. Nielepkowicz-Goździńska · A. Antczak $(\bowtie)$

Department of General and Oncological Pneumology, Medical

University of Lodz, Kopcińskiego 22, 90-153 Lodz, Poland

e-mail: adam.antczak@umed.lodz.pl

W. Fendler

Department of Pediatrics, Oncology, Hematology and

Diabetology, Medical University of Lodz, Lodz, Poland

E. Robak · L. Kulczycka-Siennicka

Department of Dermatology and Venereology, Medical

University of Lodz, Lodz, Poland

P. Górski · T. Pietras

Department of Pneumonology and Allergy, Medical University of Lodz, Lodz, Poland

E. Brzeziańska

Department of Molecular Bases of Medicine, Medical University of Lodz, Lodz, Poland compared with control group (1.00 \pm 5.99 vs. $0.00 \pm 0.56 \%, p=0.0003)$. Pulmonary fibrosis in HRCT was found in $50 \%$ of SLE patients. The disease activity scored by SLAM was significantly higher and total lung capacity was significantly lower in SLE patients with pulmonary fibrosis $\quad(8.00 \pm 3.17 \quad$ vs. $6.00 \pm 2.31$, $p=0.01 ; 88.00 \pm 28.29$ vs. $112.00 \pm 21.08 \%$ predicted, $p=0.01$, respectively). In SLE patients with pulmonary fibrosis, correlations were found between SLAM and IL-8 concentration in BALF, forced expiratory volume in $1 \mathrm{~s}$ and forced vital capacity $(r=0.65, p=0.006 ; r=-0.53$, $p=0.035 ; \quad r=-0.67, \quad p=0.006$, respectively). Our results indicate that IL-8 plays an important role in the pathogenesis of SLE. An increased concentration of IL-8 according to BALF could be considered as a useful biomarker of SLE activity and pulmonary fibrosis in SLE.

Keywords Systemic lupus erythematosus .

Interleukin-8 · Exhaled breath condensate .

Bronchoalveolar lavage fluid · Pulmonary fibrosis

\section{Introduction}

Cytokines play an important role in the process of organ damage in systemic lupus erythematosus (SLE). An increased level of cytokines was found in the serum, urine and cerebrospinal fluid of SLE patients and the concentration of these biomarkers was associated with the severity of the disease (Aringer and Smolen 2004; Dean et al. 2000). Interleukin-8 (IL-8) is suggested to play an important role both in the pathogenesis of SLE and pulmonary fibrosis (Keane and Strieter 2002). This is the first study estimating concentration of IL-8 in exhaled breath condensate (EBC) and bronchoalveolar lavage fluid (BALF) of 
SLE patients with and without pulmonary fibrosis with the aim of better understanding the degree to which IL-8 can be used as a prognostic factor in SLE patients. We have previously reported increased IL-6 and IL-10 concentrations in the BALF and IL-10 concentration in EBC in the same group of SLE patients (Nielepkowicz-Goździńska et al. 2013).

\section{Materials and Methods}

The study comprised 34 patients [fulfilling the revised American College of Rheumatology criteria for the diagnosis of SLE (Hochberg 1997)] recruited from outpatient clinics and 31 healthy controls (never-smokers members of the hospital staff and medical students with normal spirometry results) matched for sex and age. In all patients high-resolution computed tomography (HRCT), spirometry, body plethysmography, diffusion capacity for carbon monoxide corrected for hemoglobin concentration (DLCOc) measurement, EBC and BALF sample collection were performed. In all healthy individuals spirometry and EBC collection were performed, while BALF fluid collection was carried out in 20. Patients were divided into two groups, one with and one without pulmonary fibrosis in HRCT chest studies. The study was carried out with the approval of the Ethics Committee of the Medical University of Lodz (RNN/146/07/KE). A written informed consent was obtained from all patients.

Sixteen patients were taking corticosteroids (prednisone: 5-20 mg/day), three of them were treated with a combination of corticosteroids and azathioprine (100-150 mg/day), and one patient was being treated with mycophenolate mofetil (1,000 mg/day). Twelve were on a maintenance dose of immunosuppressive drugs (prednisone: $\leq 10 \mathrm{mg} /$ day, azathioprine: $\leq 100 \mathrm{mg} / \mathrm{day}$ ). Patients with a previous history of pulmonary disorders other than SLE (asthma, chronic obstructive pulmonary disease, lung cancer, pneumonia or allergies) and with current infection were excluded from the study. The disease activity was evaluated by the systemic lupus activity measure (SLAM) (Griffiths et al. 2005) and the active disease was considered as SLAM $>10$. The active stage of SLE was found in six patients, including four persons treated with immunosuppressants (Table 1).

HRCT studies were performed using a 64-slice CT (GE Healthcare LightSpeed), with $1.25 \mathrm{~mm}$ single slices, collimation at $1-\mathrm{cm}$ intervals through the lungs, $120 \mathrm{kV}$, $265 \mathrm{~mA}$, and $0.6 \mathrm{~s}$ scan time. The images were obtained at a window level of -700 Hounsfield units (HU) and a window width of 1,500 $\mathrm{HU}$ and examined by two independent experienced radiologists.

Spirometry was performed according to European Respiratory Society (ERS) standards (Miller et al. 2005).
Table 1 Demographic and clinical characteristics of SLE patients and control group

\begin{tabular}{lll}
\hline & $\begin{array}{l}\text { SLE patients } \\
(n=34)\end{array}$ & $\begin{array}{l}\text { Control group } \\
(n=31)\end{array}$ \\
\hline $\begin{array}{l}\text { Age (years) } \\
\text { Gender }\end{array}$ & $36.9 \pm 11.8$ & $31.2 \pm 4.5$ \\
Female & $31(91.2 \%)$ & $27(87.1 \%)$ \\
Male & $3(8.8 \%)$ & $4(12.9 \%)$ \\
Smoker & $4(11.8 \%)$ & $0(0 \%)$ \\
Disease duration (years) & $8.9 \pm 11.2$ & \\
SLAM score median (range) & $8(3-15)$ & \\
Active & $6(17.7 \%)$ & \\
Inactive & $28(82.3 \%)$ & \\
ANA $\geq 1: 160$ & $34(100 \%)$ & \\
P/MP therapy & $16(47 \%)$ & \\
NSAID therapy & $9(26.5 \%)$ & \\
Immunosuppressive (MM, A) therapy & $4(11.8 \%)$ & \\
\hline$A$
\end{tabular}

$A$ azathioprine, $A N A$ antinuclear antibody, $M M$ mycophenolate mofetil, MP methylprednisolone, NSAID non-steroidal anti-inflammatory drug, $P$ prednisone, $S L A M$ systemic lupus activity measure

The equipment (MES LUNGTEST 1000) was calibrated once daily. FVC and $\mathrm{FEV}_{1}$ were measured and the Tiffenau index was calculated.

Body plethysmography was obtained with a plethysmograph (Jaeger) and performed according to ERS standards (Wanger et al. 2005). Residual volume and total lung capacity (TLC) were measured.

DLCO diffusion capacity for carbon monoxide corrected for hemoglobin concentration was obtained with Lung Test 1000 Mes according to ATS/ERS guidelines (Macintyre et al. 2005).

EBC was collected by means of a condenser (Ecoscreen, Jaeger) and performed according to available recommendations (Horvath et al. 2005). After rinsing their mouth with distilled water, patients were asked to breathe out spontaneously for $10 \mathrm{~min}$ through a mouthpiece equipped with a saliva trap. A total volume of $1-3 \mathrm{ml}$ of EBC samples was collected.

BALF was collected with a flexible bronchoscope (Olympus B1-IT20) according to British Thoracic Society guidelines (2001). The tip of the bronchoscope was wedged in the lingular segments and 5 aliquots of $50 \mathrm{ml}$ of sterile $0.9 \% \mathrm{NaCl}$ solution at $37{ }^{\circ} \mathrm{C}$ were poured and recovered by gentle suction after each part. The fluid was collected, filtered through gauze and centrifuged. The pellet was suspended in phosphate buffer. Cytospins were prepared and the slides were stained with May-Grünwald Giemsa stain. The total cell count (TCC) was calculated $\left(n \times 10^{6}\right)$ under a light microscope, and the numbers of macrophages, lymphocytes, neutrophils and eosinophils were presented as percentages of the TCC. 
All samples of EBC and BALF were collected in the morning (8:00 and 10:00 a.m.) in order to avoid any influence of the circadian cycle and were immediately frozen at $-80{ }^{\circ} \mathrm{C}$ until assayed. The IL-8 level in BALF and EBC samples was measured with a commercially available enzyme-immunosorbent assay kit (Quantikine, R\&D Systems, USA) according to the manufacturer's protocols (the sensitivity $3.4 \mathrm{pg} / \mathrm{ml}$ for IL-8). The assay employs the quantitative sandwich immunoassay technique.

\section{Statistical Analysis}

The normality of distribution of continuous variables was tested using $\mathrm{W}$ test. The Student's $t$ test or Mann-
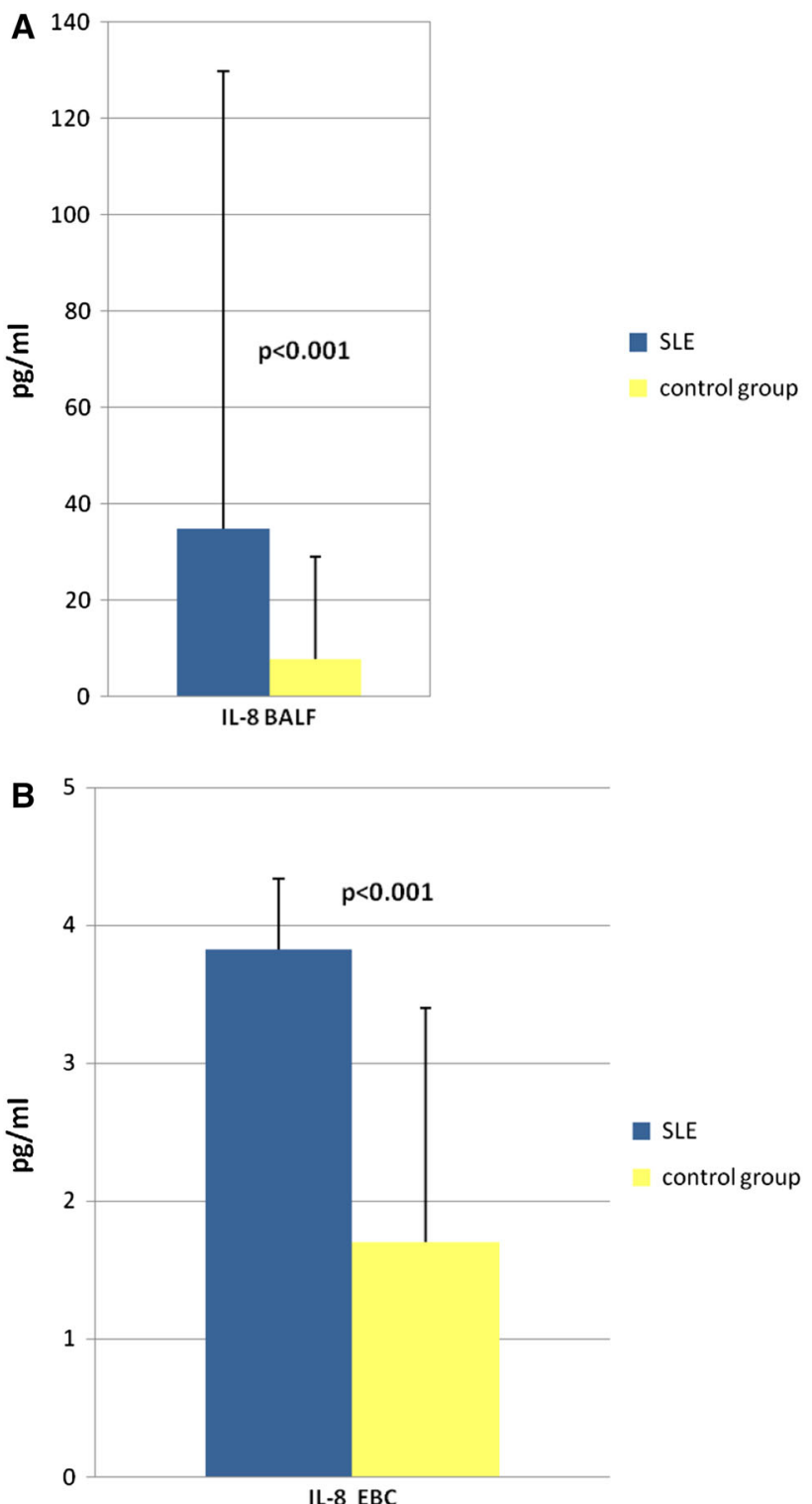

Fig. 1 IL-8 concentrations in BALF and EBC in SLE patients compared to healthy controls
Whitney's $U$ test was used for intergroup comparisons, depending on the distribution of respective variables. For comparisons of more than two groups, nonparametric analysis of variance with post hoc tests was performed using a Bonferroni-corrected Mann-Whitney's $U$ test for significant comparisons. Correlations were evaluated using the Pearson's test or Spearman's rank correlation test depending on normality of distribution. A $p$ value of $<0.05$ was assumed to be statistically significant. The STATISTICA 10.0 (StatSoft, USA) package was used for analysis.

\section{Results}

SLE patients had an increased percentage of neutrophils in BALF when compared with control group $(1.00 \pm 5.99$ vs. $0.00 \pm 0.56, p=0.0003)$. The mean IL-8 concentrations in BALF and EBC were higher in SLE patients compared to healthy controls $(34.84 \pm 95.0$ vs. $7.65 \pm 21.22 \mathrm{pg} / \mathrm{ml}$, $p<0.001 ; \quad 3.82 \pm 0.52 \mathrm{pg} / \mathrm{m} \quad$ vs. $\quad 1.7 \pm 1.7 \mathrm{pg} / \mathrm{ml}$, $p<0.001$, respectively; Fig. 1). The concentrations of

Table 2 Correlations of SLAM in SLE patients

\begin{tabular}{lrl}
\hline Correlations & $r$ value & $p$ value \\
\hline SLAM versus lymphocytes \% BALF & 0.54 & 0.001 \\
SLAM versus macrophages \% BALF & -0.52 & 0.002 \\
SLAM versus neutrophils \% BALF & 0.44 & 0.009 \\
SLAM versus eosinophils \% BALF & -0.13 & $\mathrm{~ns}(p=0.44)$ \\
SLAM versus IL-8 BALF & 0.01 & $\mathrm{~ns}(p=0.92)$ \\
SLAM versus IL-8 EBC & -0.28 & $\mathrm{~ns}(p=0.10)$ \\
SLAM versus FEV 1 /FVC (\%) & -0.03 & $\mathrm{~ns}(p=0.86)$ \\
SLAM versus FEV 1 (\%pred.) & -0.50 & 0.005 \\
SLAM versus FVC (\%pred.) & -0.57 & 0.001 \\
SLAM versus TLC (\%pred.) & -0.31 & $\mathrm{~ns}(p=0.15)$ \\
\hline
\end{tabular}

$B A L F$ bronchoalveolar lavage fluid, $E B C$ exhaled breath condensate, $F E V_{1}$ forced expiratory volume in $1 \mathrm{~s}, F V C$ forced vital capacity, $n s$ not significant, $S L A M$ systemic lupus activity measure, $T L C$ total lung capacity, \%pred. \% predicted

Table 3 HRCT positive radiological signs of pulmonary fibrosis in SLE patients

\begin{tabular}{lc}
\hline & $\begin{array}{l}\text { SLE patients } \\
(n=34)\end{array}$ \\
\hline HRCT changes & $17(50 \%)$ \\
Reticular pattern & $12(35 \%)$ \\
Areas of ground glass attenuation & $2(5.9 \%)$ \\
Interlobular interstitial thickening & $2(5.9 \%)$ \\
Air-space nodules & $3(8.8 \%)$ \\
Honeycombing & $2(5.9 \%)$ \\
\hline
\end{tabular}


Table 4 Demographic and clinical characteristics of SLE patients with and without pulmonary fibrosis

$A$ azathioprine, $A N A$ antinuclear antibody, anti-ds DNA antidouble stranded DNA, anti-RNP antibody to ribonucleoprotein, ESR erythrocyte sedimentation rate, $M M$ mycophenolate mofetil, $M P$

methylprednisolone, NSAID

non-steroidal anti-inflammatory drug, $P$ prednisone, SLAM systemic lupus activity measure

Table 5 The comparison of SLE patients with and without pulmonary fibrosis

Values are given as the mean $\pm \mathrm{SD}$

$B A L F$ bronchoalveolar lavage fluid, $D L C O c$ diffusion capacity for carbon monoxide corrected for hemoglobin concentration, $E B C$ exhaled breath condensate, $F E V_{1}$ forced expiratory volume in $1 \mathrm{~s}, F V C$ forced vital capacity, $n s$ not significant, SLAM systemic lupus activity measure, TLC total lung capacity, \%pred. \% predicted

\begin{tabular}{|c|c|c|}
\hline & $\begin{array}{l}\text { SLE with pulmonary } \\
\text { fibrosis }(n=17)\end{array}$ & $\begin{array}{l}\text { SLE without pulmonary } \\
\text { fibrosis }(n=17)\end{array}$ \\
\hline Age $($ mean \pm SD) & $36.1 \pm 11.2$ & $37.2 \pm 11.5$ \\
\hline Smoker & $2(11.8 \%)$ & $2(11.8 \%)$ \\
\hline \multicolumn{3}{|l|}{ Gender } \\
\hline Female & $16(94.1 \%)$ & $15(88.2 \%)$ \\
\hline Male & $1(5.9 \%)$ & $2(11.8 \%)$ \\
\hline SLAM score median (range) & $8.00 \pm 3.17$ & $6.00 \pm 2.31$ \\
\hline Active & $5(29.4 \%)$ & $1(5.9 \%)$ \\
\hline Inactive & $12(70.6 \%)$ & $16(94.1 \%)$ \\
\hline ANA $\geq 1: 160$ & $17(100 \%)$ & $17(100 \%)$ \\
\hline Positive anti-dsDNA & $2(11.8 \%)$ & $2(11.8 \%)$ \\
\hline Anti-RNP & $0(0 \%)$ & $0(0 \%)$ \\
\hline P/MP therapy & $8(47 \%)$ & $8(47 \%)$ \\
\hline NSAID therapy & $5(29.4 \%)$ & $4(23.5 \%)$ \\
\hline Immunosuppressive (MM, A) therapy & $2(11.8 \%)$ & $2(11.8 \%)$ \\
\hline Fever & $1(5.9 \%)$ & $2(11.8 \%)$ \\
\hline Skin and mucous membrane lesion & $9(52.9 \%)$ & $6(35.3 \%)$ \\
\hline Arthritis & $7(41.2 \%)$ & $4(23.5 \%)$ \\
\hline Renal active disorders & $2(11.8 \%)$ & $1(5.9 \%)$ \\
\hline Cardiac disorders & $1(5.9 \%)$ & $0(0 \%)$ \\
\hline Neuropsychiatric disorders & $6(35.3 \%)$ & $5(29.4 \%)$ \\
\hline Gastrointestinal disorders & $4(23.5 \%)$ & $2(11.8 \%)$ \\
\hline Lymphadenopathy & $0(0 \%)$ & $0(0 \%)$ \\
\hline Anemia $(\mathrm{Hb}<12 \mathrm{~g} / \mathrm{dl})$ & $3(17.6 \%)$ & $3(17.6)$ \\
\hline Leucopenia (WBC <3.5 cc mm) & $5(29.4 \%)$ & $2(11.8 \%)$ \\
\hline Thrombocytopenia $(<150,000 \mathrm{cc} \mathrm{mm})$ & $2(11.8 \%)$ & $1(5.9 \%)$ \\
\hline $\mathrm{ESR}>25 \mathrm{~mm} / \mathrm{h}$ & $6(35.3 \%)$ & $3(17.6 \%)$ \\
\hline
\end{tabular}

\begin{tabular}{lccl}
\hline Parameter & $\begin{array}{l}\text { SLE with pulmonary } \\
\text { fibrosis }(n=17)\end{array}$ & $\begin{array}{l}\text { SLE without pulmonary } \\
\text { fibrosis }(n=17)\end{array}$ & $p$ value \\
\hline SLAM & $8.00 \pm 3.17$ & $6.00 \pm 2.31$ & $p=0.01$ \\
Time of SLE duration (years) & $11.00 \pm 8.16$ & $5.00 \pm 6.33$ & $\mathrm{~ns}(p=0.42)$ \\
Lymphocytes \% BALF & $20.82 \pm 12.27$ & $16.00 \pm 8.27$ & $\mathrm{~ns}(p=0.29)$ \\
Eosinophils \% BALF & $0.00 \pm 1.94$ & $1.00 \pm 1.3$ & $\mathrm{~ns}(p=0.07)$ \\
Neutrophils \% BALF & $2.00 \pm 8.02$ & $1.00 \pm 2.02$ & $\mathrm{~ns}(p=0.37)$ \\
Macrophages \% BALF & $81.00 \pm 14.84$ & $80.00 \pm 8.04$ & $\mathrm{~ns}(p=0.19)$ \\
FEV 1 /FVC (\%) & $82.37 \pm 8.14$ & $85.50 \pm 4.94$ & $\mathrm{~ns}(p=0.25)$ \\
FEV $(\%$ pred.) & $90.50 \pm 21.09$ & $100.00 \pm 16.59$ & $\mathrm{~ns}(p=0.09)$ \\
FVC (\%pred.) & $93.26 \pm 22.44$ & $106.00 \pm 15.09$ & $\mathrm{~ns}(p=0.27)$ \\
TLC (\%pred.) & $88.00 \pm 28.29$ & $112.00 \pm 21.08$ & $p=0.01$ \\
DLCOc $(\%$ pred.) & $90.00 \pm 28.32$ & $98.00 \pm 15.72$ & $\mathrm{~ns}(p=0.43)$ \\
IL-8 BALF (pg/ml) & $41.3 \pm 59.48$ & $28.06 \pm 121.23$ & $\mathrm{~ns}(p=0.42)$ \\
IL-8 EBC (pg/ml) & $3.92 \pm 0.39$ & $3.78 \pm 0.63$ & $\mathrm{~ns}(p=0.89)$ \\
\hline
\end{tabular}

IL-8 in the EBC of all healthy persons were below the detection limit and were arbitrarily assumed to be half of the detection limit value.
In the group of SLE patients, SLAM was correlated with the percentage of neutrophils, lymphocytes and macrophages in BALF $(r=0.44, \quad p=0.009 ; \quad r=0.54$, 
Table 6 The comparison of SLE patients in relation to immunosuppressive treatment

Values are given as the mean $\pm \mathrm{SD}$

$B A L F$ bronchoalveolar lavage fluid, $D L C O c$ diffusion capacity for carbon monoxide corrected for hemoglobin concentration; $E B C$ exhaled breath condensate, $F E V_{1}$ forced expiratory volume in $1 \mathrm{~s}, F V C$ forced vital capacity, $n s$ not significant, SLAM systemic lupus activity measure, $T L C$ total lung capacity, \%pred. \% predicted

\begin{tabular}{|c|c|c|c|}
\hline Parameter & $\begin{array}{l}\text { With immunosuppressive } \\
\text { treatment }(n=16)\end{array}$ & $\begin{array}{l}\text { Without immunosuppressive } \\
\text { treatment }(n=18)\end{array}$ & $p$ value \\
\hline SLAM & $8.00 \pm 3.87$ & $7.00 \pm 1.90$ & $\mathrm{~ns}(p=0.35)$ \\
\hline Time of SLE duration (years) & $6.00 \pm 6.27$ & $8.00 \pm 8.49$ & ns $(p=0.63)$ \\
\hline Lymphocytes \% BALF & $16.00 \pm 10.30$ & $17.00 \pm 10.10$ & $\mathrm{~ns}(p=0.79)$ \\
\hline Eosinophils \% BALF & $0.00 \pm 0.89$ & $1.00 \pm 2.09$ & $\mathrm{~ns}(p=0.052)$ \\
\hline Neutrophils \% BALF & $2.50 \pm 7.82$ & $1.0 \pm 3.77$ & $\mathrm{~ns}(p=0.41)$ \\
\hline Macrophages \% BALF & $80.00 \pm 11.13$ & $82.00 \pm 13.41$ & $\mathrm{~ns}(p=0.81)$ \\
\hline $\mathrm{FEV}_{1} / \mathrm{FVC}(\%)$ & $82.20 \pm 6.96$ & $85.00 \pm 6.69$ & $\mathrm{~ns}(p=0.29)$ \\
\hline $\mathrm{FEV}_{1}$ (\%pred.) & $90.13 \pm 25.25$ & $100.00 \pm 12.76$ & $\mathrm{~ns}(p=0.29)$ \\
\hline FVC (\%pred.) & $97.50 \pm 25.13$ & $107.00 \pm 12.73$ & $\mathrm{~ns}(p=0.53)$ \\
\hline TLC (\%pred.) & $88.50 \pm 36.35$ & $97.00 \pm 11.82$ & $\mathrm{~ns}(p=0.31)$ \\
\hline DLCOc (\%pred.) & $99.00 \pm 31.37$ & $90.00 \pm 13.38$ & ns $(p=0.14)$ \\
\hline IL-8 BALF (pg/ml) & $49.42 \pm 135.00$ & $29.63 \pm 18.07$ & ns $(p=0.07)$ \\
\hline IL-8 EBC (pg/ml) & $3.82 \pm 0.42$ & $3.82 \pm 0.63$ & ns $(p=0.64)$ \\
\hline
\end{tabular}

$p=0.001 ; \quad r=-0.52, \quad p=0.002$, respectively). The activity of the disease correlated negatively with $\mathrm{FEV}_{1}$ and FVC $(r=-0.50, \quad p=0.005 ; \quad r=-0.57, \quad p=0.001$, respectively; Table 2$)$.

Seventeen patients (50\%) were found to have pulmonary fibrosis in HRCT (Table 3). The disease activity scored by SLAM was significantly higher and TLC lower in the SLE patients with pulmonary fibrosis when compared with patients with normal HRCT $(8.00 \pm 3.17$ vs. $6.00 \pm 2.31 ; 88.00 \pm 28.29$ vs. $112.00 \pm 21.08 \%$ predicted, $p=0.01$ in both). Demographic and clinical characteristics of SLE patients with and without pulmonary fibrosis are found in Table 4. The concentration of IL-8 in BALF and EBC, the spirometric results $\left(\mathrm{FEV}_{1} / \mathrm{FVC}, \mathrm{FEV}_{1}\right.$, FVC, DLCO) and the numbers of macrophages, lymphocytes, neutrophils and eosinophils in BALF were not significantly different in the patients with pulmonary fibrosis when compared with patients without pulmonary fibrosis (Table 5). Also there were no differences in the concentration of IL-8, the lung function tests or SLAM in SLE patients associated with immunosuppressive treatment (Table 6). However, among SLE patients with pulmonary fibrosis IL-8 concentration in BALF was significantly higher, $\mathrm{FEV}_{1}$ and $\mathrm{FVC}$ were significantly lower in patients with immunosuppressive treatment when compared with patients without immunosuppressive treatment (81.38 \pm 81.23 vs. $29.61 \pm 19.14, p<0.05 ; 73.37 \pm 18.67$ vs. $101.75 \pm 12.03, p<0.01 ; 78.71 \pm 23.76$ vs. $106.00 \pm$ 11.25, $p<0.05$, respectively; Fig. 2; Table 7). SLAM was found to have negative correlations with $\mathrm{FEV}_{1}$ and FVC $(r=-0.53, p=0.035 ; r=-0.67, p=0.006)$ and positive correlation with IL-8 concentration in BALF ( $r=$ $0.65, p=0.006)$ in patients with pulmonary fibrosis (Fig. 3).

\section{Discussion}

In the present study, the percentage of neutrophils in BALF was found to be higher in SLE patients when compared with healthy individuals and the findings are in accordance with previous research (BAL Cooperative Group Steering Committee 1990; Groen et al. 1993; Witt et al. 1996). Wallaert et al. (1990) reported lymphocytic predominance in the BAL fluid from lupus patients and impaired function of alveolar macrophages. They also proposed that subclinical alveolitis is present in SLE, with an accumulation of inflammatory and immune cells in the BAL fluid in patients without clinical and radiological respiratory abnormalities. These results indicate the presence of a cellmediated immune response in the respiratory system of lupus patients.

Diffuse lung disease during lung biopsy and at autopsy has been observed in 4-70\% of SLE patients (Haupt et al. 1981). Interstitial lung disease is a relatively uncommon complication of SLE but the pulmonary changes attributable to interstitial abnormalities are found in CT scans of 30-38 \% SLE patients with normal chest radiographs (Kakati et al. 2007; Keane and Lynch 2000). Additionally, HRCT reveals interstitial abnormalities in SLE patients with normal pulmonary function test results (Fenlon et al. 1996). Our results confirm a high occurrence of fibrotic changes in the lungs of SLE patients and the coexistence of fibrotic changes with normal lung function of lupus patients.

There is a growing body of evidence that chemokines are involved in the pathogenesis of diffuse lung disease. IL8 is one of the major mediators of the inflammatory response and leads to neutrophil recruitment in the pulmonary interstitium and airspace. CXCL8 can activate a 

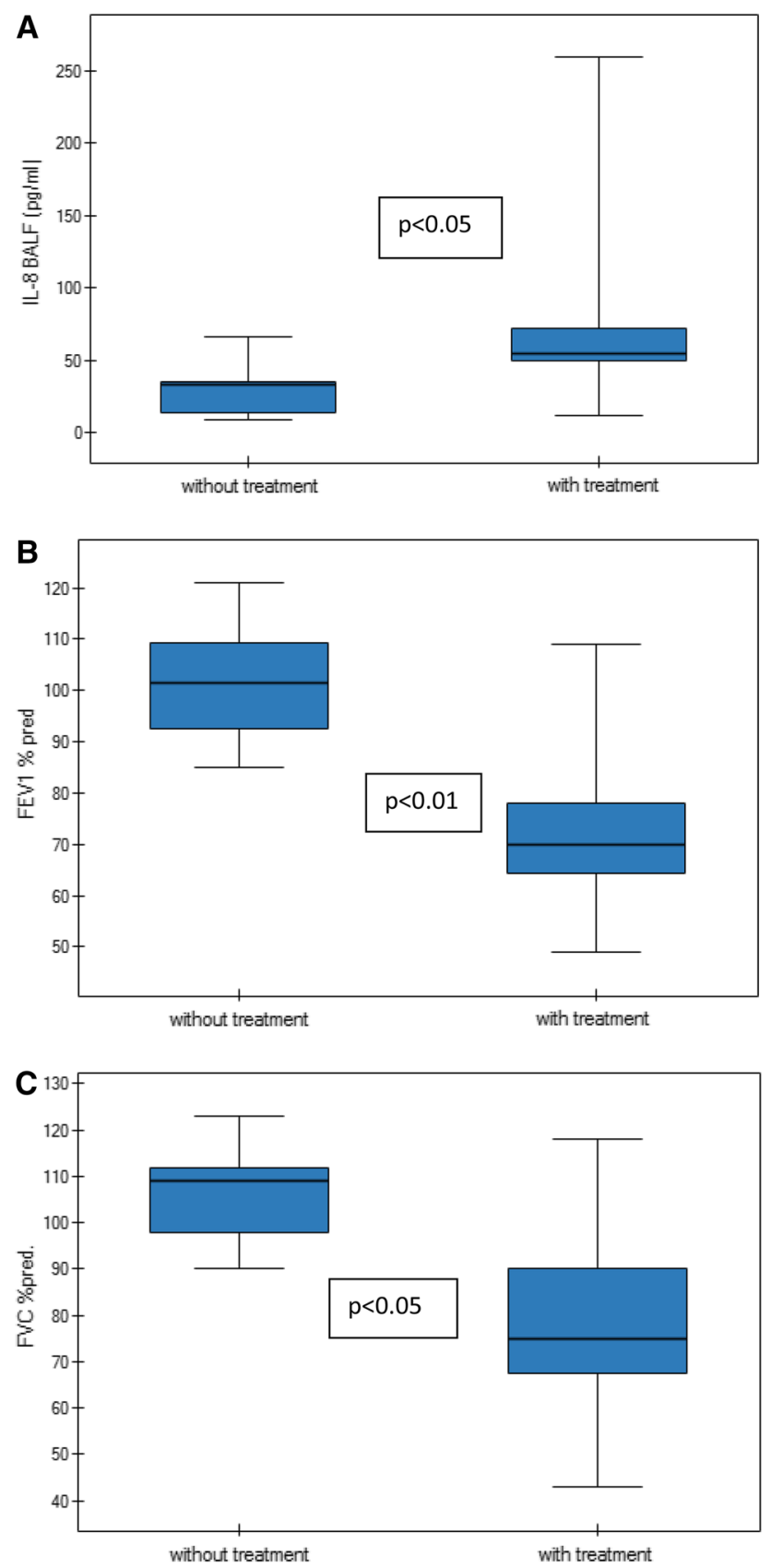

Fig. 2 The comparison of IL-8 level in BALF, FEV ${ }_{1}$ and FVC of SLE patients with pulmonary fibrosis in relation to immunosuppressive treatment

wide range of neutrophil functions including degranulation and respiratory burst (Knall et al. 1996). Sustained neutrophil accumulation in the alveolar space (neutrophilic alveolitis) causes lung injury and leads to interstitial fibrosis (Lynch et al. 1992; Pantelidis et al. 1997). Increased levels of IL- 8 have been described in many inflammatory disorders characterized by neutrophilic infiltration, including psoriasis and rheumatoid arthritis (Biasi et al. 1998; Endo et al. 1991). The fact that IL-8 is a chemotactic cytokine which recruits the inflammatory cells to a particular location could make it a parameter of localized inflammation.

Al-Mutairi et al. (2007) report that lupus patients with pulmonary involvement have higher levels of pro-inflammatory cytokines in serum than those without pulmonary involvement. Lit et al. (2006) report a positive correlation of IL-8 plasma level with SLEDAI (systemic lupus erythematosus disease activity index in SLE patients). In the present study, the level of IL- 8 was significantly higher in BALF and EBC of SLE patients. No significant differences were found in the numbers of particular BALF cells or IL-8 concentrations between patients with and without pulmonary fibrosis, despite the fact that SLAM correlates with IL-8 level in the BALF of patients with fibrotic changes in HRCT. We hypothesize that the lack of differences between patients with and without pulmonary fibrosis may be an effect of the influence of other factors like cytokines (tumor necrosis factor, IL-10), immunosuppressive therapy or the predominance of non-active SLE patients in the study. The immunosuppressive therapy is a limiting factor for the study accuracy.

An increased IL-8 level in BALF and serum of systemic sclerosis patients has been reported (Bolster et al. 1997; Furuse et al. 2003; Meloni et al. 2004). Moreover, Furuse et al. (2003) detected that elevated serum IL-8 concentrations significantly correlated with decreased percentage of DLCO in systemic sclerosis patients. They suggested that the elevation of chemokines correlates with pulmonary involvements.

The level of IL-8 mRNA in the idiopathic pulmonary fibrosis (IPF) patients correlated with the number of neutrophils in BALF and with the degree of disease severity (Carre et al. 1991). Southcott et al. (1995) suggested that the levels of IL-8 in IPF may correlate with poor prognosis.

In our study SLE patients with pulmonary fibrosis and immunosuppressive treatment had higher IL-8 concentration in BALF when compared with patients with pulmonary fibrosis and without immunosuppressive treatment. Thomas et al. (2002) observed that steroids (dexamethasone) inhibited IL-8 secretion from peripheral blood mononuclear cells, but on the other hand the inhibitory effects of steroids on IL-8 secretion were absent in infants and they developed more severe bronchiolitis. In the present study, the immunosuppressive therapy altered the results and is a limiting factor for the study. The inhibitory effects of steroids on IL-8 secretion in SLE need to be studied further.

In conclusion, to the best of our knowledge, this is the first study estimating the concentration of IL-8 in the EBC and BALF of SLE patients with and without pulmonary fibrosis. Our observations confirm the importance of IL-8 
Table 7 The comparison of SLE patients with pulmonary fibrosis in relation to immunosuppressive treatment

Values are given as the mean $\pm \mathrm{SD}$

$B A L F$ bronchoalveolar lavage fluid, $D L C O c$ diffusion capacity for carbon monoxide corrected for hemoglobin concentration; $E B C$ exhaled breath condensate, $F E V_{1}$ forced expiratory volume in $1 \mathrm{~s}, F V C$ forced vital capacity, $n s$ not significant, SLAM systemic lupus activity measure, $T L C$ total lung capacity, \%pred. \% predicted

\begin{tabular}{lccl}
\hline Parameter & $\begin{array}{c}\text { With immunosuppressive } \\
\text { treatment }(n=16)\end{array}$ & $\begin{array}{c}\text { Without immunosuppressive } \\
\text { treatment }(n=18)\end{array}$ & $p$ value \\
\hline SLAM & $10.75 \pm 3.88$ & $7.55 \pm 1.23$ & $\mathrm{~ns}$ \\
Time of SLE duration (years) & $12.0 \pm 6.48$ & $8.57 \pm 9.50$ & $\mathrm{~ns}$ \\
Lymphocytes \% BALF & $18.50 \pm 12.72$ & $22.88 \pm 12.23$ & $\mathrm{~ns}$ \\
Eosinophils \% BALF & $1.44 \pm 2.55$ & $0.12 \pm 0.35$ & $\mathrm{~ns}$ \\
Neutrophils \% BALF & $7.37 \pm 10.37$ & $3.11 \pm 4.98$ & $\mathrm{~ns}$ \\
Macrophages \% BALF & $77.42 \pm 14.19$ & $72.22 \pm 15.77$ & $\mathrm{~ns}$ \\
FEV 1 /FVC (\%) & $79.12 \pm 7.18$ & $85.62 \pm 8.15$ & $\mathrm{~ns}$ \\
FVC (\%pred.) & $78.71 \pm 23.76$ & $106.00 \pm 11.25$ & $p<0.05$ \\
FEV $1 \%$ pred.) & $73.37 \pm 18.67$ & $101.75 \pm 12.03$ & $p<0.01$ \\
TLC (\%pred.) & $81.71 \pm 16.34$ & $97.50 \pm 13.18$ & $\mathrm{~ns}(p=0.052)$ \\
DLCOc (\%pred.) & $98.87 \pm 20.06$ & $87.77 \pm 13.85$ & $\mathrm{~ns}$ \\
IL-8 BALF (pg/ml) & $81.38 \pm 81.23$ & $29.61 \pm 19.14$ & $p<0.05$ \\
IL-8 EBC (pg/ml) & $3.76 \pm 0.44$ & $3.87 \pm 0.38$ & $\mathrm{~ns}$ \\
\hline
\end{tabular}

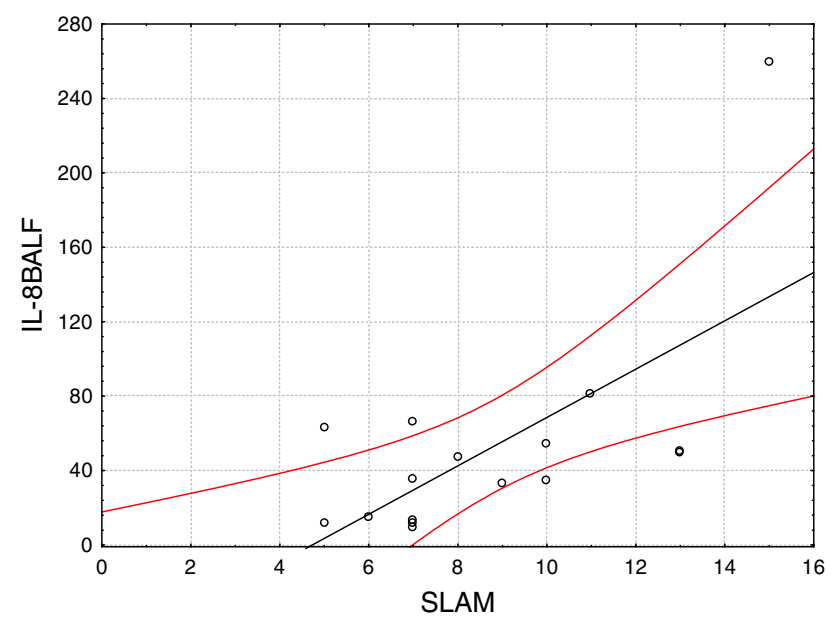

Fig. 3 Positive correlation of SLAM with IL-8 concentration in BALF in SLE patients with pulmonary fibrosis $(r=0.65, p=0.006)$

in the pathogenesis of SLE. The increased concentration of IL-8 in BALF could be considered as a marker of pulmonary fibrosis in SLE. The decision whether to start treatment is often the most difficult clinical challenge, because many patients have limited pulmonary fibrosis that will not necessarily progress. Immunosuppressive drugs could be administered to patients with an increased level of IL-8 in BALF. IL-8 in BALF may be a valuable biomarker to monitor disease activity in SLE patients with pulmonary fibrosis. IL-8 could also be used as a potential therapeutic target of pulmonary fibrosis in SLE. Further studies are necessary for the evaluation of the prognostic role of these biomarkers in the EBC and BALF of SLE patients and the inhibitory effects of steroids on IL-8 secretion in SLE patients.
Acknowledgments The study was funded from the Polish Ministry of Science and Higher Education grant no. N N402 292336.

Conflict of interest None declared.

Open Access This article is distributed under the terms of the Creative Commons Attribution License which permits any use, distribution, and reproduction in any medium, provided the original author(s) and the source are credited.

\section{References}

Al-Mutairi S, Al-Awadhi A, Raghupathy R et al (2007) Lupus patients with pulmonary involvement have a pro-inflammatory cytokines profile. Rheumatol Int 27:621-630

Aringer M, Smolen JS (2004) Tumour necrosis factor and other proinflamatory cytokines in systemic lupus erythematosus: a rationale for therapeutic intervention. Lupus 13:344-347

BAL Cooperative Group Steering Committee (1990) Bronchoalveolar lavage constituents in healthy individuals, idiopathic pulmonary fibrosis, and selected comparison groups. Am Rev Respir Dis 141(5Pt 2):169-202

Biasi D, Carletto A, Caramaschi P et al (1998) Neutrophil functions and IL-8 in psoriatic arthritis and in cutaneous psoriasis. Inflammation 22:533-543

Bolster MB, Ludwicka A, Sutherland SE et al (1997) Cytokine concentrations in bronchoalveolar lavage fluid of patients with systemic sclerosis. Arthr Rheum 40:743-751

British Thoracic Society Bronchoscopy Guidelines Committee, a Subcommittee of the Standards of Care Committee of the British Thoracic Society (2001) British Thoracic Society guidelines on diagnostic flexible bronchoscopy. Thorax 56(Suppl 1):i1-i21

Carre PC, Mortenson RL, King TE Jr et al (1991) Increased expression of the interleukin- 8 gene by alveolar macrophages in idiopathic pulmonary fibrosis a potential mechanism for the recruitment and activation of neutrophils in lung fibrosis. J Clin Invest 88:1802-1810

Dean G, Tyrrell-Price J, Crawley E et al (2000) Cytokines and systemic lupus erythematosus. Ann Rheum Dis 59:243-251 
Endo H, Akahoshi T, Takagishi K et al (1991) Elevation of interleukin-8 (IL-8) levels in joint fluids of patients with rheumatoid arthritis and the induction by IL-8 of leukocyte infiltration and synovitis in rabbit joints. Lymphokine Cytokine Res 10:245-252

Fenlon HM, Doran M, Sant SM et al (1996) High-resolution chest CT in systemic lupus erythematosu. AJR Am J Roentgenol 166: 301-307

Furuse S, Fujii H, Kaburagi Y et al (2003) Serum concentrations of the CXC chemokines interleukin 8 and growth-regulated oncogene-alpha are elevated in patients with systemic sclerosis. J Rheumatol 30:1524-1528

Griffiths B, Mosca M, Gordon C (2005) Assessment of patients with systemic lupus erythematosus and the use of lupus disease activity indices. Best Pract Res Clin Rheumatol 19:685-708

Groen H, Aslander M, Bootsma H et al (1993) Bronchoalveolar lavage cell analysis and lung function impairment in patients with systemic lupus erythematosus (SLE). Clin Exp Immunol 94:127-133

Haupt HM, Moore GW, Hutchins GM (1981) The lung in systemic lupus erythematosus: analysis of the pathologic changes in 120 patients. Am J Med 71:791-798

Hochberg MC (1997) Updating the American College of Rheumatology revised criteria for the classification of systemic lupus erythematosus. Arthr Rheum 40:1725

Horvath I, Hunt J, Barnes PJ et al (2005) Exhaled breath condensate: methodological recommendations and unresolved questions. Eur Respir J 26:523-548

Kakati S, Doley B, Dihingia P et al (2007) A clinical study of pulmonary manifestations in systemic lupus erythematosus with special reference to CT findings. Indian J Rheumatol 12:133-136

Keane MP, Lynch JP III (2000) Pleuropulmonary manifestations of systemic lupus erythematosus. Thorax 55:159-166

Keane MP, Strieter RM (2002) The importance of balanced proinflammatory and anti-inflammatory mechanisms in diffuse lung disease. Respir Res 3:5

Knall C, Young S, Nick JA et al (1996) Interleukin-8 regulation of the Ras/Raf/mitogenactivated protein kinase pathway in human neutrophils. J Biol Chem 271:2832-2838
Lit LC, Wong CK, Tam LS et al (2006) Raised plasma concentration and ex vivo production of inflammatory chemokines in patients with systemic lupus erythematosus. Ann Rheum Dis 65:209-215

Lynch JP III, Standiford TJ, Rolfe MW et al (1992) Neutrophilic alveolitis in idiopathic pulmonary fibrosis: the role of interleukin-8. Am J Respir Crit Care Med 145:1433-1439

Macintyre N, Crapo RO, Viegi G et al (2005) Standardization of the single-breath determination of carbon monoxide uptake in the lung. Eur Respir J 26:720-735

Meloni F, Caporali R, Marone Bianco A et al (2004) Cytokine profile of bronchoalveolar lavage in systemic sclerosis with interstitial lung disease: comparison with usual interstitial pneumonia. Ann Rheum Dis 63:892-894

Miller MR, Hankinson J, Brusasco V et al (2005) Standardisation of spirometry. Eur Respir J 26:319-338

Nielepkowicz-Goździńska A, Fendler W, Robak E et al (2013) Exhaled cytokines in systemic lupus erythematosus with lung involvements. Pol Arch Med Wewn 123:141-148

Pantelidis P, Southcott AM, Black CM et al (1997) Up-regulation of IL-8 secretion by alveolar macrophages from patients with fibrosis alveolitis: a subpopulation analysis. Clin Exp Immunol 108:95-104

Southcott AM, Jones KP, Li D et al (1995) Interleukin-8, differential expression in lone fibrosing alveolitis and systemic sclerosis. Am J Respir Crit Care Med 151:1604-1612

Thomas LH, Sharland M, Friedland JS (2002) Steroids fail to downregulate respiratory syncytial virus-induced IL-8 secretion in infants. Pediatr Res 52:368-372

Wallaert B, Dugas M, Dansin E et al (1990) Subclinical alveolitis in immunological systemic disorders. Transition between health and disease? Eur Respir J 3:1206-1216

Wanger J, Clausen JL, Coates A et al (2005) Standardisation of the measurement of lung volumes. Eur Respir J 26:511-522

Witt C, Dörner T, Hiepe F et al (1996) Diagnosis of alveolitis in interstitial lung manifestation in connective tissue diseases: importance of late inspiratory crackles, 67 gallium scan and bronchoalveolar lavage. Lupus 5:606-612 